\title{
Viral Vector Systems for Gene Therapy: A Comprehensive Literature Review of Progress and Biosafety Challenges
}

Applied Biosafety:

Journal of ABSA International 2020, Vol. 25(I) 7-18

(C) ABSA International 2020

Article reuse guidelines:

sagepub.com/journals-permissions DOI: 10.1 I $77 / / 535676019899502$ journals.sagepub.com/home/apb

(QSAGE

\author{
Sumit Ghosh' ${ }^{\oplus}$, Alex M. Brown', Chris Jenkins ${ }^{2}$, and Katie Campbell'
}

\begin{abstract}
Introduction: National Institutes of Health $(\mathrm{NIH})$ defines gene therapy as an experimental technique that uses genes to treat or prevent disease. Although gene therapy is a promising treatment option for a number of diseases (including inherited disorders, some types of cancer, and certain viral infections), the technique remains risky and is still under study to make sure that it will be effective and safe.

Methods: Applications of viral vectors and nonviral gene delivery systems have found an encouraging new beginning in gene therapy in recent years. Although several viral vectors and nonviral gene delivery systems have been developed in the past 3 decades, no one delivery system can be applied in gene therapy to all cell types in vitro and in vivo. Furthermore, the use of viral vector systems (both in vitro and in vivo) present unique occupational health and safety challenges. In this review article, we discuss the biosafety challenges and the current framework of risk assessment for working with the viral vector systems.

Discussion: The recent advances in the field of gene therapy is exciting, but it is important for scientists, institutional biosafety committees, and biosafety officers to safeguard public trust in the use of this technology in clinical trials and make conscious efforts to engage the public through ongoing forums and discussions.
\end{abstract}

\section{Keywords}

gene therapy, risk assessment, viral vectors, occupational health, biosafety

\section{Introduction}

Gene therapy involves altering the genes inside your body's cells in an attempt to treat or stop disease. ${ }^{1}$ Genes contain your DNA that code for much of your body's form and function, from making you grow taller to regulating your body systems. ${ }^{2}$ Genes that do not work properly can cause disease. Gene therapy replaces a faulty gene or adds a new gene in an attempt to cure disease or improve your body's ability to fight disease. ${ }^{3-5}$ This powerful technology is currently used to treat or improve the health condition of the patient by modifying the patient's cells genetically. ${ }^{6,7}$ It provides a unique approach to address both inherited and acquired diseases by delivering a therapeutic gene material and its associated regulatory elements into the nucleus to correct the loss of function caused by mutation or express the deficient gene product at physiologic levels. ${ }^{8-12}$ The use of this technology requires assessing health and environmental risks and further stresses the importance of biosafety governance in risk assessment and mitigation. ${ }^{5,13-18}$

In the present day, both preclinical and clinical settings have used both viral and nonviral vectors in innovative modifications and applications. ${ }^{1,19}$ These vector systems offer the potential for efficient gene delivery or can act as a vital tool or vehicle to treat genetic disease. ${ }^{2,20,21}$ As of 2019, almost
2600 gene therapy clinical trial studies have been completed, are ongoing, or have been approved worldwide. ${ }^{22,23}$ Furthermore, 232 gene and cell therapy trials have been approved worldwide, and 4 gene therapy products that were approved by the Food and Drug Administration (FDA) are available in the United States. ${ }^{15,22,23}$ The majority of these gene therapy trials, almost $70 \%$, are based on viral vectors. ${ }^{15,24}$ Although transfer of genetic material into cells may be accompanied through nonviral vector-based delivery systems, we will be evaluating only the most common biosafety issues associated with viral-based vector systems in this review.

Viral vectors have generally proved efficient tools for gene delivery to target cells/tissue, a critical aspect of achieving therapeutic efficacy. ${ }^{25}$ Furthermore, viral vector expression cassettes can be engineered with elements to enhance target

\footnotetext{
' The Abigail Wexner Research Institute at Nationwide Children's Hospital, Columbus, OH, USA

${ }^{2}$ Clinical Biosafety Services, A Division of Sabai Global, Wildwood, MO, USA

\section{Corresponding Author:}

Sumit Ghosh, The Abigail Wexner Research Institute at Nationwide Children's Hospital, Columbus, OH 43215, USA.

Email: Sumit.Ghosh@NationwideChildrens.org
} 
specificity and increase transgene expression. ${ }^{12,26}$ These features have been explored and exploited to develop efficient methods for the delivery of genes of interest into mammalian cells. However, a vast repertoire of viral vectors exists with profoundly different properties. To date, 5 main classes of viral vectors have been tested for clinical applications. ${ }^{25,27}$ These include retroviruses (RV), adenoviruses (AV), adenoassociated viruses (AAV), lentiviruses (LV), and herpes simplex viruses (HSV). ${ }^{8,28}$ Although many viral vectors are safe and effective delivery vehicles for clinical gene therapy, some viral vectors are considered risky for potential oncogenesis. ${ }^{3,29}$ Furthermore, some viral vectors (eg, Adenovirus vector, Herpesviral vector) are toxic and can be immunogenic. ${ }^{22}$

Using viral vector systems for gene therapy are promising treatment options for several diseases. However, these methods remain risky and are still under study to ensure safety and efficacy during clinical trials. Additionally, there are limited resources available to institutional biosafety committees (IBCs) and health care providers when it comes to risk assessment and developing procedures to minimize risk and exposure in a research/clinical setting. This review article focuses on the viral vector systems utilized for gene therapy and some of the biosafety challenges associated with each given that there are no consensus guidelines for working with them in a research/ clinical setting because it is dictated by risk assessment.

\section{Viral Vectors}

The 5 main classes of viral vectors can be categorized in 2 groups according to whether their genomes integrate into host cellular chromatin (oncoretroviruses and LV) or persist in the cell nucleus predominantly as extrachromosomal episomes (AAV, AV, and herpes viruses) ${ }^{6,30}$ The choice of viral vectors for clinical use depends on the efficiency of transgene expression, ease of production, safety, toxicity, and stability. ${ }^{31}$ Furthermore, the types of vectors are represented by both RNA and DNA viruses with either single-stranded (ss) or doublestranded (ds) genomes. ${ }^{8}$ The National Institutes of Health (NIH) Recombinant DNA Guidelines and the World Health Organization (WHO) Laboratory Biosafety Manual categorize infectious agents into risk groups for laboratory research (Risk Groups 1-4). ${ }^{23}$ The risk group informs about the level of containment needed to minimize risk during the handling of these infectious agents as designated by its biosafety level. ${ }^{23}$ The Centers for Disease Control and Prevention (CDC), WHO, and $\mathrm{NIH}$ have published recommendations for work at different biosafety levels (BSL-1 to BSL-4) for wild-type virus and viral vector handling in a laboratory research setting. When viral vector systems are used in gene therapy experiments in a clinical setting, these recommendations are adapted to do a comprehensive risk assessment and for developing a mitigation plan. ${ }^{19,23}$ As with risk group classification, biosafety levels differ by viral vector systems. Historically, IBCs have been responsible for reviewing any risks associated with viral shedding and insertional mutagenesis during the protocol review process. ${ }^{32}$ The main groups of viral vectors applied for gene therapy and their recommended risk group/biosafety level are summarized in Table 1.

\section{Adeno-Associated Virus Vectors}

Adeno-associated virus vectors are nonpathogenic parvoviruses, consisting of a linear single-stranded DNA (ssDNA) genome of approximately 4.7 kilobases (kb), with two 145 nucleotide-long inverted terminal repeats (ITRs) at the termini. ${ }^{33,34}$ The virus does not encode a polymerase and therefore relies on cellular polymerases for genome replication. ${ }^{19}$ The AAV genome consists of 3, open-reading frames (ORF) flanked by ITRs. ${ }^{35}$ The ITRs function as the viral origin of replication (rep) and the packaging signal (cap). ${ }^{33}$ The rep ORF encodes 4 nonstructural proteins. These structural proteins play a role in viral replication, transcriptional regulation, genomic integration, and virion assembly. ${ }^{36}$ The cap ORF encodes 3 structural proteins (VP1, VP2, and VP3) that assemble to form a 60 -mer (repeat unit) viral capsid. ${ }^{37}$ Finally, an ORF that is present as an alternate reading frame within the cap gene produces the assembly-activating protein, a viral protein that localizes AAV capsid proteins to the nucleolus and functions in the capsid assembly process. ${ }^{38}$ In recombinant forms of $\mathrm{AAV}$, the gene of interest is inserted between the ITRs in place of rep and cap, and the latter is provided in trans, along with helper viral genes, during vector production. ${ }^{39}$ The significant advantage is that the resulting vector can transduce both dividing and nondividing cells, with stable transgene expression in the absence of the helper virus in postmitotic tissue..$^{28,38,40}$ There are 11 naturally occurring serotypes and more than 100 variants of AAV that differ in amino acid sequence, particularly within the hypervariable regions of the capsid proteins. ${ }^{1,38}$ As a result, these variants of AAV vary somewhat in their gene delivery properties, which is utilized successfully in several gene therapy applications. ${ }^{41,42}$

$\mathrm{AAV}$ is one of the most common vectors used in gene therapy. ${ }^{19}$ One of the primary reasons for using AAV is that AAV has a long-term and efficient transgene expression in various cell types in many tissues such as liver, muscle, retina, and the central nervous system (CNS). ${ }^{43-45}$ However, the application of AAV has some associated disadvantages. The preexisting immunity to human AAV vectors is comparable to the AV, and the integration into the host genome (if it occurs at all) is random, which can lead to accidental activation or inhibition of endogenous gene expression. ${ }^{46}$ Another substantial limitation of AAV vectors is their small packaging capacity that is generally considered to be $<5 \mathrm{~kb} .{ }^{19}$ This limitation makes AAV vector design for diseases involving larger genes challenging or impossible. ${ }^{38,47}$ Additionally, various AAV serotypes have exhibited remarkably different expression patterns due to differences in cell entry and intracellular activities. ${ }^{48}$ For instance, AAV1 is suitable for expression in skeletal muscle and the retina, ${ }^{49,50}$ whereas AAV5 transduces neuronal and lung cells efficiently. ${ }^{51,52}$ By contrast, since AAV2 has long-term, albeit poor, expression levels, the discovery of novel rhesus monkey serotypes AAV7 and AAV8 might be of interest for human 


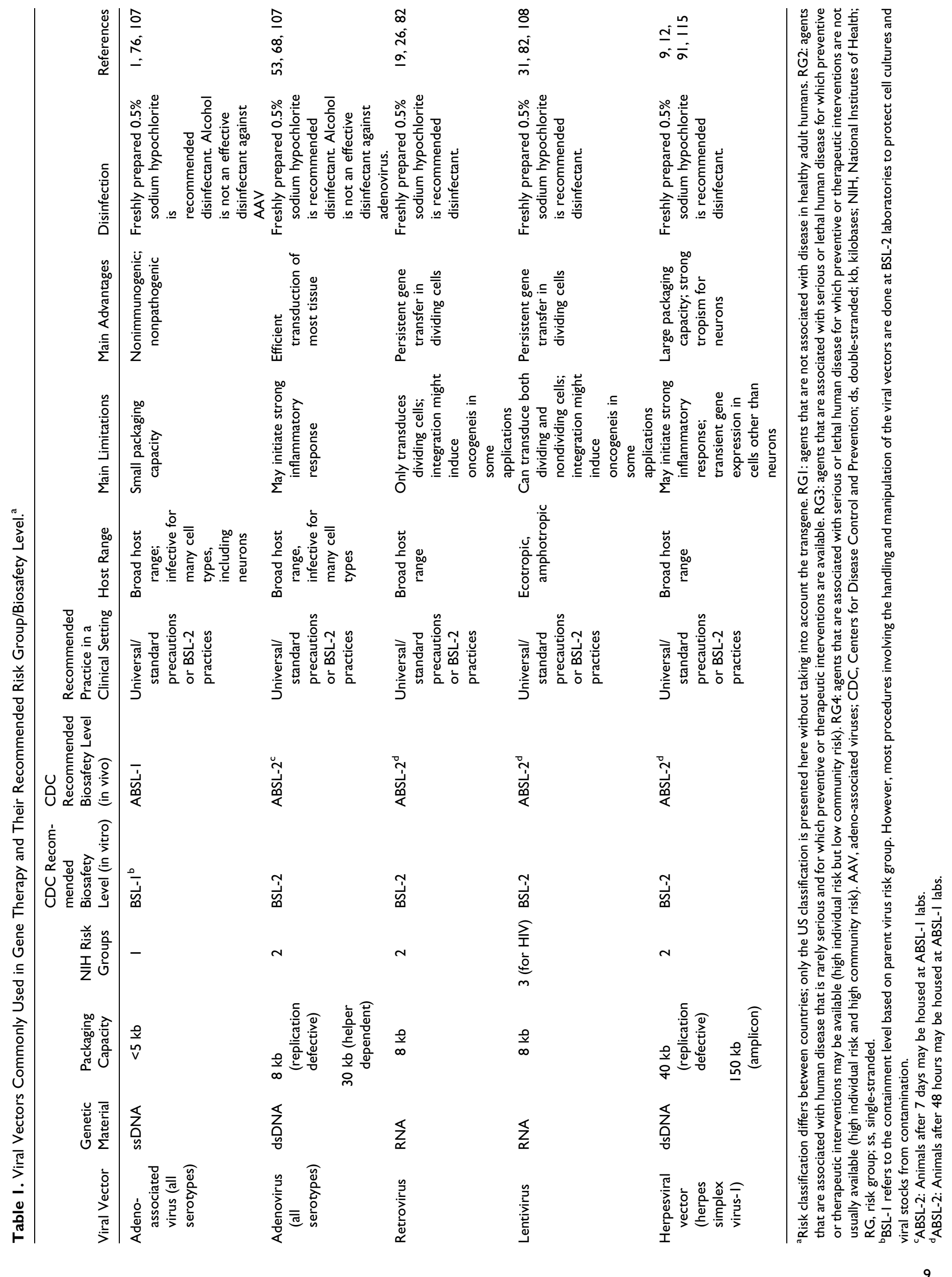


gene therapy applications. ${ }^{53,54}$ In particular, AAV8 demonstrated higher levels (up to 100-fold higher) of factor IX expression in liver cells than for any other AAV serotype tested. ${ }^{55}$ Moreover, preimmunization with different AAV serotypes did not compromise the expression levels. ${ }^{55}$

AAV vectors have an excellent safety record in clinical trials and preclinical animal studies. ${ }^{2,56}$ AAV is generally handled at BSL-1 ${ }^{57}$ In a clinical setting, universal precautions or standard precautions are utilized while working with AAV vectors. Exceptions include synthetic or recombinant AAV constructs created in the presence of a helper virus and those that contain a potentially harmful transgene. ${ }^{58}$ While AAV can infect humans and other primate species, AAV is not known to cause disease. ${ }^{19}$ Approximately $80 \%$ of humans are seropositive to AAV strains. ${ }^{40}$ However, repeated administrations, which can trigger an immune response, may be a limiting factor. ${ }^{59}$ Applying a different AAV serotype for each readministration typically alleviates this reaction. ${ }^{60}$ Moreover, several gene clinical trials have revealed that AAV vectors do not stimulate a heightened immune response. ${ }^{60}$ In some animal models, the integration of recombinant AAV increased the incidence of tumor formation, but this association has not been observed in humans. ${ }^{61}$ Although ineffective in replication as an isolated agent, AAV has the potential for replication competence in the presence of a helper plasmid or virus, and stock solutions should be tested for the presence of other agents. ${ }^{62}$

\section{Adenovirus Vectors}

Adenoviruses are a class of DNA viruses with a doublestranded $34 \mathrm{~kb}$ to $43 \mathrm{~kb}$ genome, which employs alternative splicing to encode genes in both the sense and antisense orientations. ${ }^{63}$ The AV genome is flanked by 2 ITRs and contains 8 transcription units. ${ }^{64}$ The early regions (E1A, E1B, E2, E3, and E4) are the first regions transcribed and encode proteins involved in activating transcription of other viral regions and altering the cellular environment to promote viral production. ${ }^{64}$ The late regions (L1-L5) are transcribed from an alternatively spliced transcript. ${ }^{65}$ Similar to recombinant AAV vectors, the $\mathrm{AV}$ genome remains in an extrachromosomal form following infection. ${ }^{66}$ Humans have 51 different serotypes of AV; $45 \%$ to $80 \%$ of the population harbors neutralizing antibodies against Ad5, the most prevalent, due to natural infections, which typically date back to infancy. ${ }^{67}$ Standard recombinant adenoviral vectors have the E1 unit deleted, but the removal of E3 can create vectors capable of packaging up to $7.5 \mathrm{~kb}$ of foreign DNA. ${ }^{68}$ Vectors with both E1 and E4 units deleted result in lower immune responses. ${ }^{69}$ Finally, in helper-dependent, or gutless, adenoviral vectors, all adenoviral genes are deleted, and a helper virus provides adenoviral genes for production. ${ }^{70}$ Although these adenoviral genes have a carrying capacity of more than $30 \mathrm{~kb}$, they are currently challenging to manufacture. ${ }^{71}$ Another concern with adenoviral vectors, in general, is that the particles themselves elicit cellular inflammatory responses as opposed to relatively inert AAV virions. ${ }^{72}$
Compared with other viral gene delivery systems, adenoviral vectors offer significant advantages. First, the AV is the most effective means of delivering genes in vivo as most human cells express the primary AV receptor and the secondary integrin receptors. ${ }^{73}$ Thus, human cells are easily infected with AV vectors and consequently yield high levels of the transgene expression. Second, the development of gutless adenoviral vectors allows us to circumvent antiadenoviral vector immunity. ${ }^{63}$ The AV vector now represents 1 of the 3 major viral vector categories in the gene therapy toolbox, along with $\mathrm{AAV}$ and lenti/retrovirus vector systems. ${ }^{19,22}$ Interestingly, some of the inherited shortcomings of AV, such as immunity evoked against the AV capsid and low-level expression of AV genes, may now prove beneficial for the development of anticancer immunotherapies, where inducing immunity against cancer or directly killing the cancer cell is the goal. ${ }^{74,75}$ The combined immunity against the $\mathrm{AV}$ and the short time of expression may prove beneficial for using AV to develop potential vaccines. ${ }^{76,77}$

Work with AV vectors is done using BSL-2 practices. ${ }^{78}$ In a clinical setting, universal precautions or standard precautions are utilized while working with AV vectors. ${ }^{23}$ Although AV cause mild respiratory disease or conjunctivitis in healthy adults, they are notoriously immunogenic. ${ }^{6}$ Several episodes of inflammatory responses to AV vectors in clinical trials have been documented, including 1 death after direct injection of an adenoviral vector into the hepatic artery. ${ }^{79}$ Some firstgeneration adenoviral vectors, which retain a considerable proportion of the genome, have initiated dose-dependent apoptosis of various target cells in vitro and in multiple mouse models in vivo, indicating a potential for direct cytotoxicity. ${ }^{24,80}$ The widespread distribution and tropism of AV support the potential for intracellular recombination or complementation of the vector with previously acquired wild-type AV in either packaging cell lines in vivo, thus making the adenoviral vector replication competent. ${ }^{81}$ This risk can be reduced substantially by using cell lines with decreased or no homology between vector and helper sequences. Another risk-reduction strategy is regular quality control checks of viral vector stocks for possible low levels of contamination with the replication-competent virus. Currently, researchers have determined these threshold levels arbitrarily because no standards govern thresholds to date. Further improvements regarding reactions with the immune system, viral longevity, vector packaging capacity, and contamination will be pursued as we continue making breakthroughs using adenoviral vectors in gene therapy.

\section{Retrovirus Vectors}

Retroviruses are relatively complex enveloped RNA viruses with a diploid ssRNA genome and consist of at least 4 genes, gag, pro, pol, and env. ${ }^{30,82}$ The gag gene encodes the primary structural polyprotein, which is necessary for the assembly of noninfectious and immature viral-like particles. ${ }^{2}$ The pro gene encodes the viral protease and is responsible for facilitating the maturation of viral particles. ${ }^{19}$ Products of the pol gene include 
reverse transcriptase, $\mathrm{RNase} \mathrm{H}$, and integrase, whereas env is responsible for the viral surface glycoprotein and transmembrane proteins that mediate cellular receptor binding and membrane fusion. ${ }^{83}$ The typical feature of RV and retroviral vectors is their ability to integrate into host DNA. ${ }^{78}$ Besides, complex RV such as HIV-1 encode accessory proteins that enhance replication and infectivity. ${ }^{84}$ Viral RNA is reversibly transcribed and integrated into the form of a provirus. ${ }^{84}$ The RV very effectively cooperate with enzymes of the host cell, which they use for their replication and long-term expression of viral proteins ${ }^{85}$ Like most viruses, the entry of the RV into the host cell is receptor-dependent. ${ }^{83}$ Several of the oncogenic RV are replication-defective forms that have substituted a part of their standard viral gene complement with an oncogene sequence. ${ }^{86}$ Replication-competent retroviruses also cause malignant disease and a range of other pathogenic states in a wide variety of species. ${ }^{87}$ The acquired immunodeficiency syndrome (AIDS) is caused by the retroviruses HIV-1 and HIV- $2 .{ }^{88}$

Retroviral vectors have been extensively used to deliver therapeutic genes in the context of gene therapy, clinical applications for monogenic disorders, cancer, and infectious diseases, providing a stable and efficient expression of the transgene to patients. ${ }^{30,89-91}$ Retroviruses have numerous advantages over other vectors. The most significant advantage that retroviral vectors offer is their ability to transform their ssRNA genome into a dsDNA molecule that stably integrates into the target cell genome. ${ }^{85}$ This feature enables the retroviral vectors to modify the host cell nuclear genome permanently. Currently, retroviral-vector-mediated gene transfer has been reinvigorated with the development of a new retroviral vectors class derived from $\mathrm{LV} .{ }^{8}$ The LV have the unique ability among $\mathrm{RV}$ to infect noncycling cells. ${ }^{92}$ Vectors derived from LV have provided a substantial leap in gene-editing technology and present new avenues to achieve significant levels of gene transfer in vivo. ${ }^{19}$

Containment levels for the use of retroviruses are determined based on the cell types they infect. BSL-1 is appropriate for RV that do not infect human cells. BSL-2 is necessary if they are used to infect human cells. ${ }^{56}$ The primary safety risk with the use of RV arises from their ability to integrate into the host cell chromosome, which raises the possibility of insertional mutagenesis and oncogene activation. ${ }^{93}$ Generation of replication-competent RV in target cells or tissues is the primary risk related to the use of retroviral vectors. ${ }^{94}$ Assessment of this risk is critical in determining the safety associated with the use of retroviral vector systems.

Furthermore, the target cell range of the vector is also a safety issue. Incorporation of a virus envelope that can infect cells from multiple species increases both the risk of generating replication-competent retroviruses and the potential danger of any resulting virus, which could spread from one species to another. ${ }^{30,94}$ Future studies that utilize retroviral vectors in gene therapy experiments must explore safer strategies that focus on the risks associated with in vivo recombination, generation of mosaic RV, and long-term storage of viral genetic information.

\section{Lentivirus Vectors}

Lentiviruses, a type of RV, consist of a single-stranded positive-sense RNA sequence that is transcribed into a DNA and integrated into the host genome, causing persistent infection. ${ }^{95}$ Most lentiviral vectors (LVVs) are derived from HIV-1 and retain the capability to integrate into the genome of infected cells. ${ }^{96}$ Wild-type HIV genome with all of its genes and regulatory elements provides the backbone for LVVs. ${ }^{96} \mathrm{In}$ the first-generation LVVs, the envelope protein and the $p s i(\psi)$ packaging signal are removed and incorporated into a heterologous promoter to reduce recombination potential. ${ }^{97}$ The second-generation LVVs have the accessory genes (vif, vpr, $v p u$, and nef) removed to reduce the virulence of any potential replication-competent retrovirus. ${ }^{97}$ Additionally, the thirdgeneration LVVs have the transactivator gene, tat, eliminated, which splits the vector into 3 plasmids to reduce further recombination potential, retaining only the 3 genes necessary for transgene expression (gag, pol, rev). ${ }^{97}$ Fourth-generation LVVs split the gag and pol onto separate plasmids to reduce even further recombination potential. ${ }^{90}$ This generation also added back some HIV genes to enhance transduction efficiency and transgene expression. ${ }^{98}$

Lentiviral vectors have become very popular for clinical applications due to their ability to more efficiently transduce nonproliferating or slowly proliferating cells, such as CD34 ${ }^{+}$stem cells. ${ }^{99}$ Gene transfer using LVVs into $\mathrm{CD} 34^{+}$HSCs has been used to treat several genetic diseases, including $\beta$-thalassemia, ${ }^{100} \mathrm{X}$-linked adrenoleukodystrophy, ${ }^{101}$ metachromatic leukodystrophy, ${ }^{102}$ and WiskottAldrich Syndrome. ${ }^{103}$ Recently, multiple clinical trials used third-generation, self-inactivating LVVs to introduce genes into hematopoietic stem cells to correct primary immunodeficiencies and hemoglobinopathies. ${ }^{104}$ The LVVs have been used to alter $T$ cells by introducing genes to generate immunity to fight cancer through the delivery of chimeric antigen receptors (CARs) or cloned T-cell receptors. ${ }^{105}$ CAR T-cell therapies developed using LVVs have been successful in the clinical setting in patients with B-cell malignancies. This has resulted in the regulatory approval of the first genetically engineered cellular therapy using these vector systems. ${ }^{106}$

The use of LVVs in research is still associated with potential risks, and the long-term safety of these clinical interventions is still being evaluated. While lentiviral systems are derived from HIV, their organization across numerous plasmids and the deletion of many HIV proteins lowers the likelihood of generating replication-capable virus. ${ }^{85,107}$ These vector systems are handled at BSL-2. The limitations of using LVVs in clinical trials today are mainly due to insufficient methods for the production of high-titer virus stocks and the safety concerns related to their origin from HIV, despite the engineering of packaging cell lines and deletions of genes required for viral replication. ${ }^{90,108}$ One approach to address safety issues has been to develop LVVs incapable of replication in human cells. Although LVVs are less associated with insertional 
mutagenesis than other RV, these vectors still provide evidence of off-target effects. ${ }^{109}$

\section{Herpesviral Vectors}

Herpes simplex virus is an enveloped virus with a dsDNA genome more than $150 \mathrm{~kb}$ in length, which is divided into long and short unique segments $\left(\mathrm{U}_{\mathrm{L}}\right.$ and $\mathrm{U}_{\mathrm{S}}$, respectively) and flanked by inverted repeated sequences $\left(\mathrm{TR}_{\mathrm{L}} / \mathrm{IR}_{\mathrm{L}}\right.$ and $\mathrm{TR}_{\mathrm{S}} /$ $\left.\mathrm{IR}_{\mathrm{S}}\right){ }^{110}$ The HSV genome encodes approximately 90 genes, almost half of which are nonessential and can be eliminated in recombinant vectors. ${ }^{111}$ Currently, gene delivery and gene therapy applications use 3 types of HSV vectors. ${ }^{111}$ First, amplicons are gene delivery systems packaged through transfection into producer cells of plasmids containing the HSV origin of DNA replication, HSV cleavage-packaging sequences, and transgene(s) of interest. ${ }^{47}$ The plasmids form head-to-tail linked concatemers that are packaged into the viral particles, which can, in general, accommodate large fragments of foreign DNA. ${ }^{47}$ In a second system, the deletion of genes necessary for the lytic cycle of HSV can create less toxic, replication-defective vectors that elicit a smaller immune response than wild-type HSV vectors. ${ }^{112}$ Deleting various combinations of the immediate, early genes that otherwise lead to the expression of the HSV infected cell proteins (ICP0, ICP4, ICP22, ICP27, and ICP47) creates several versions of replication-defective HSV vectors. ${ }^{12}$ Finally, replicationcompetent HSV vectors are attenuated versions of HSV that contain the genes required for replication in vitro but lack the genes necessary for replication in vivo. ${ }^{113}$ Replicationcompetent vectors serve as oncolytic therapies and vaccines, where the set of deleted genes must lead to the appropriate balance of replicative attenuation for safety and lytic activity for tumor toxicity or vaccine immunogenicity, respectively. ${ }^{113}$

$\mathrm{HSV}-1$ is a neurotropic virus that displays numerous necessary adaptations to the nervous system. With regard to the neurological application, the design of gene therapy vectors can exploit each adaptation rationally. ${ }^{114} \mathrm{HSV}$ vectors containing latency-active promoters LAP1 and LAP2 have been engineered for long-term expression (up to 300 days) in the peripheral nervous system. ${ }^{114}$ HSV vectors have also found applications in the CNS. ${ }^{115}$ However, because wild-type HSV causes encephalitis, developing nontoxic replication-defective vectors with deletions in the IE genes (ICP4, ICP22, ICP27) ${ }^{114}$ is essential. Furthermore, recent breakthroughs in the field of HSV-1 technology authorize and support the use of HSV-1 as vaccine vectors for the delivery of foreign antigens. ${ }^{116}$ In particular, HSV vectors exhibit several advantages for prophylaxis against viral infections. ${ }^{115}$

HSV is transmitted by direct contact and mucous membrane exposure to body excretions and respiratory droplets. ${ }^{113}$ Although molecular alterations to the viral genome have dramatically reduced virus-associated risks, HSV remains a known human pathogen with inflammatory and immunomodulatory effects. BSL-2 is appropriate for many constructs of HSV. ${ }^{19}$ Despite high seroprevalence of both HSV1 and HSV2 in the United States (approximately 53\% and 16\%, respectively), few robust data that support in vivo recombination or complementation are available. ${ }^{70}$ However, fatal meningoencephalitis can occur, especially in immunocompromised persons. ${ }^{113}$

\section{Recommendations for Policies and Procedure Development for Comprehensive Risk Assessment for Gene Therapy Research Utilizing Viral Vector Systems}

Safe use of viral vector systems in human gene therapy research is a major concern for most IBCs in a research/clinical setting because there is no single document that summarizes the guidelines of working with them. The NIH Recombinant DNA guidelines and the fifth edition of Biosafety in Microbiological and Biomedical Laboratories (BMBL) offer the general framework of working with viral vectors, and they are often used as a reference for risk assessment for human gene transfer research. ${ }^{23}$ However, there is minimal information about conducting a comprehensive risk assessment when using these vectors systems or FDA-approved gene therapy products in a health care research setting. As a result, it becomes very challenging for IBCs across organizations to develop a common framework for protocol review of gene therapy research by interpreting the recommendations outlined in $B M B L$ and $\mathrm{NIH}$ Recombinant DNA guidelines. Additionally, institutional policies govern how the organization institutional review board (IRB) interacts with IBCs during the human gene transfer research protocol review process.

The IRB review is necessary to protect the rights and welfare of research subjects in clinical investigations. ${ }^{117}$ The IRB requires a comprehensive review and approval for any research with human subjects supported by the U.S. Department of Health and Human Services (HHS) or regulated by FDA. ${ }^{118,119}$ The IRB review is necessary for research supported or conducted by any of the federal agencies subscribing to the Common Rule, for research on products regulated by FDA, and for research conducted by investigators at any institution giving Federalwide Assurance (eg, universities assuring the federal government that all of their research will conform to federal rules on human subjects research). ${ }^{120,121}$ An IRB is also responsible for approving amendments to a study and review a progress report at least yearly. ${ }^{122} \mathrm{An}$ IRB has the authority to approve, require modifications to (as a condition of approval), or deny approval to gene therapy research and informedconsent documents. ${ }^{119}$ Additionally, an IRB has the authority to terminate or suspend any study for noncompliance. ${ }^{123}$ The revised NIH Guidelines (April 2019) eliminate the RAC review, protocol registration, and reporting requirements associated with gene therapy research. ${ }^{124}$ In addition, a frequently asked question blog on the NIH website outlines the separation of the IBC with the IRB during the human gene therapy protocol review process. ${ }^{125}$ The revised NIH Guidelines highlight the critical role of IBCs in reviewing gene therapy research studies. ${ }^{124}$ IBCs are now mandated by the NIH Guidelines, 


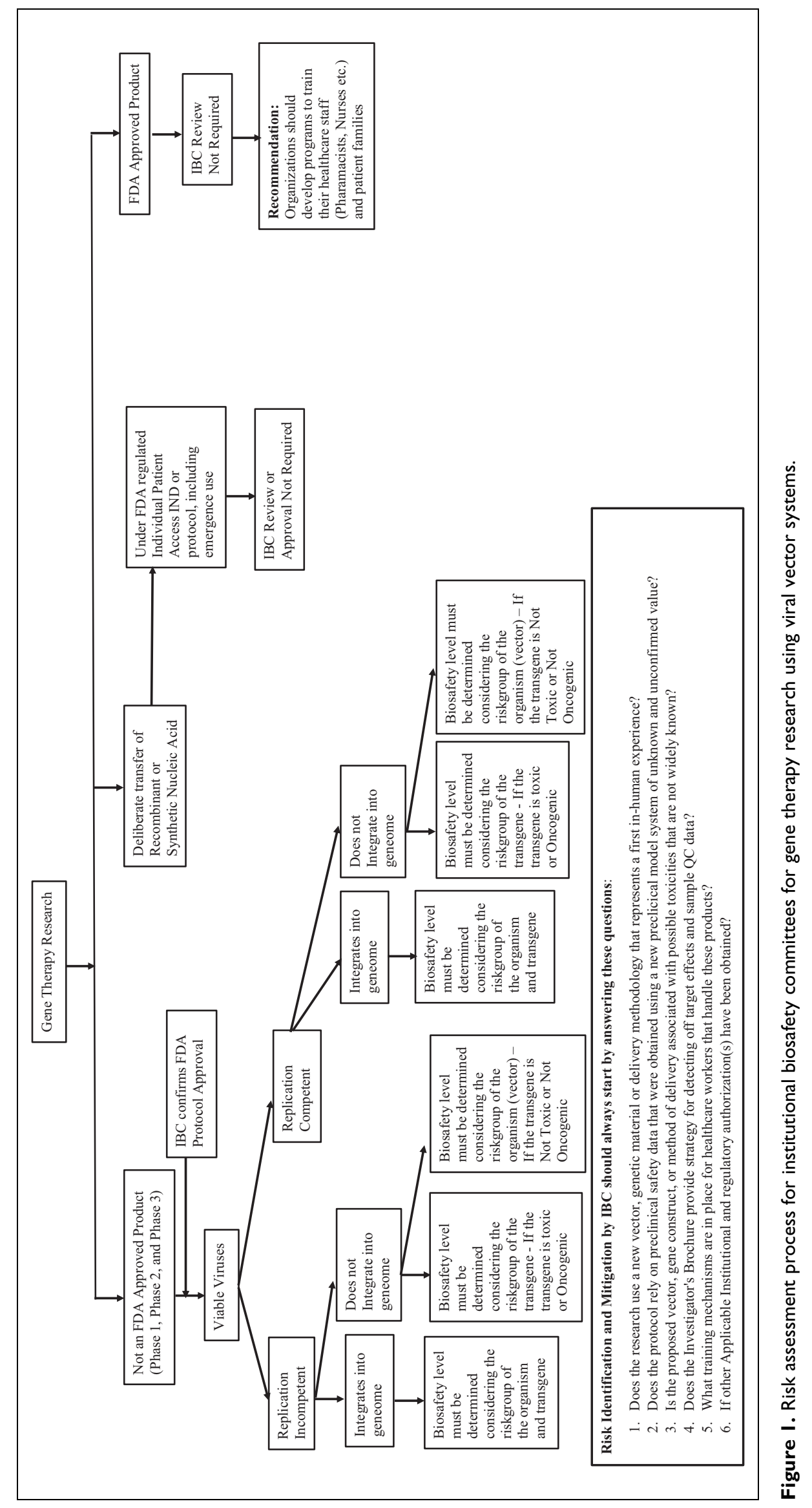


Section III-C, to undertake a review of safety risks at the level of the investigators' institutions (eg, universities and research centers) for all forms of human gene therapy research. ${ }^{124}$ This highlights the need for IRBs to collaborate with institutional IBCs during the protocol risk assessment review process. As IBCs play a critical role in providing oversight of gene therapy research, it is recommended that institutional compliance committees (eg, IRB, IBCs) play a significant role in ensuring compliance with education, training, risk assessment, and policy development of research involving human gene therapy.

During the protocol review of human gene therapy research, the IBCs play a critical role in reviewing risks associated with viral shedding. Shedding in the field of gene therapy means dissemination of the gene therapy product through the excreta of the treated patient or subject. ${ }^{126}$ Despite the substantial number of clinical gene therapy trials that have been conducted worldwide, there is currently no summary of actual shedding data available for different viral vector systems. There have been studies that have shown that some of the vectors used in gene therapy research can be excreted from the body for a least a week after injection. ${ }^{32}$ This shedding of the vector can be found in the blood, urine, saliva, and stool for up to a week following injection. ${ }^{127}$ However, there are limited peerreviewed publications that outline shedding data of different viral vector systems when administered to human research subjects. Most of the Investigator Brochures and also the product insert of ZOLGENSMA recommend 30-day universal or standard precautions. ${ }^{126,128}$ In general, most health care facilities recommend universal/standard precautions with patient material between 14 and 30 days after administration for both health care staff and direct family members, dependent on viral shedding analysis. ${ }^{128}$ Additionally, instructions are provided to family members and caregivers to practice good hand hygiene for a minimum of 2 weeks after the injection. ${ }^{128}$ This requires washing hands with soap regularly and using appropriate protective gloves if coming into direct contact with patients' bodily fluids and waste. ${ }^{128}$ This provides an insight into the occurrence of shedding of vectors in clinical practice and can offer support for environmental risk assessment in future clinical gene therapy applications using different viral vector systems so that a consensus criteria can be developed. Some recommendations for the Risk Assessment process for IBCs for gene therapy research using Viral Vector Systems is summarized in Figure 1.

Luxturna (Spark Therapeutics), the first virally delivered gene therapy, was approved in December 2017. ${ }^{129}$ In August 2017, the FDA also approved the use of Tisagenlecleucel, marketed as Kymriah (Novartis), which is a treatment for B-cell acute lymphoblastic leukemia. ${ }^{130}$ Additionally, in October 2017, Yescarta (Kite Pharma Inc/Gilead) became the first CAR $\mathrm{T}$ cell therapy approved by the FDA for use in adult patients. ${ }^{23}$ Most recently in May 2019, the FDA approved Onasemnogene abeparvovec, sold under the trade name Zolgensma, a gene therapy medication to treat spinal muscular atrophy in children. ${ }^{131}$ As more gene therapy products are getting approved by the FDA, future research should focus on developing biosafety recommendations and policies for health care personnel (physicians, nurses, fellows, pharmacists, etc) handing gene therapy products that should encompass the following: storage, cleaning, preparation, dispensing, accidental spillage/exposure, personal protective clothing, equipment (eg, biosafety cabinets-type), disinfection and decontamination procedures, transport, and disposal of waste. Additionally, a framework for training health care workers and families of patients using these products should be developed.

\section{Conclusions}

In this review, we highlight some of the biosafety challenges associated with the current viral vector systems that have applications in gene therapy research. Current advancements in the field of gene therapy have opened the door to cures at a molecular level for many genetic diseases. The design of new experimental viral vectors and emerging technologies highlight the critical role of biosafety professionals in managing gene therapy research protocols, identifying risk level, and developing acceptable policies predominantly in the absence of consensus framework for the risk assessment process.

\section{Acknowledgment}

We thank Melody Davis at The Abigail Wexner Research Institute at Nationwide Children's Hospital for critical reading of the manuscript.

\section{Ethical Approval Statement}

Not applicable to this study.

\section{Statement of Human and Animal Rights}

Not applicable to this study.

\section{Statement of Informed Consent}

Not applicable to this study.

\section{Declaration of Conflicting Interests}

The authors declared no potential conflicts of interest with respect to the research, authorship, and/or publication of this article.

\section{Disclaimer}

The views presented in this publication are those of the authors and do not necessarily reflect the position of the associated institutions.

\section{Funding}

The authors received no financial support for the research, authorship, and/or publication of this article.

\section{ORCID iD}

Sumit Ghosh (D) https://orcid.org/0000-0002-6890-954X 


\section{References}

1. Boulaiz H, Marchal JA, Prados J, Melguizo C, Aránega A. Nonviral and viral vectors for gene therapy. Cell Mol Biol (Noisy-legrand). 2005;51(1):3-22.

2. Kootstra NA, Verma IM. Gene therapy with viral vectors. Annu Rev Pharmacol Toxicol. 2003;43:413-439.

3. Fears R, Ter Meulen V. Assessing security implications of genome editing: emerging points from an international workshop. Front Bioeng Biotechnol. 2018;6:34.

4. Lee C. CRISPR/Cas9-based antiviral strategy: current status and the potential challenge. Molecules. 2019;24(7):E1349.

5. Simón JE, Rodríguez ÁS, Santiago Vispo N. CRISPR-Cas9: a precise approach to genome engineering. Applied Biosafety. 2018;52(6):701-707.

6. Lundstrom K. Viral vectors in gene therapy. Diseases. 2018;6(2): E42.

7. Guedon JM, Wu S, Zheng X. Current gene therapy using viral vectors for chronic pain. Mol Pain. 2015;11:27.

8. Hansen JE, Gram GJ. [Viral vectors for clinical gene therapy]. Ugeskr Laeger. 2002;164(37):4272-4276.

9. Strayer DS. Viral vectors for gene therapy: past, present and future. Drug News Perspect. 1998;11(5):277-286.

10. Safary A, Akbarzadeh Khiavi M, Mousavi R, Barar J, Rafi MA. Enzyme replacement therapies: what is the best option? Bioimpacts. 2018;8(3):153-157.

11. Wraith JE. Limitations of enzyme replacement therapy: current and future. J Inherit Metab Dis. 2006;29(2-3):442-447.

12. Marshall E. Gene therapy. viral vectors still pack surprises. Science. 2001;294(5547):1640.

13. Chapman JE, Gillum D, Kiani S. Approaches to reduce CRISPR off-target effects for safer genome editing. Applied Biosafety. 2017;22(1):7-13.

14. Birnbaum JE. Adaptive risk management of gene drive experiments: biosafety, biosecurity, and ethics. Applied Biosafety. 2017; 22(3):97-103.

15. Eisenman D. The United States' regulatory environment is evolving to accommodate a coming boom in gene therapy research. Applied Biosafety. 2019;24(3):147-152.

16. Heitman E, James KS, Collins P. Gene drives on the horizon: issues for biosafety. Applied Biosafety. 2016;21(4).

17. Jennifer E. Chapman DG, Kiani S. Approaches to reduce CRISPR OFF-target effects for safer genome editing. Applied Biosafety. 2017;22(1):7-13.

18. Krishnan P GD. Gene Drive 101: a basic guidance resource for biosafety professionals. Applied Biosafety. 2017;22(4): 181-184.

19. Chen YH, Keiser MS, Davidson BL. Viral vectors for gene transfer. Curr Protoc Mouse Biol. 2018;8(4):e58.

20. Hutchinson E. American Society of Gene Therapy-first annual meeting. Education session: the ABCs of non-viral vectors for gene therapy. 28-31 May 1998, Seattle, Washington, USA. IDrugs. 1998;1(3):265-267.

21. Shah PB, Losordo DW. Non-viral vectors for gene therapy: clinical trials in cardiovascular disease. Adv Genet. 2005;54:339-361.
22. Ginn SL, Amaya AK, Alexander IE, Edelstein M, Abedi MR. Gene therapy clinical trials worldwide to 2017: an update. J Gene Med. 2018;20(5):e3015.

23. Petrich J, Marchese D, Jenkins C, Storey M, Blind J. Gene replacement therapy: a primer for the health-system pharmacist [published online June 27, 2019]. J Pharm Pract. doi:10.1177/ 0897190019854962

24. Lee CS, Bishop ES, Zhang R, et al. Adenovirus-mediated gene delivery: potential applications for gene and cell-based therapies in the new era of personalized medicine. Genes Dis. 2017;4(2): 43-63.

25. Powell SK, Rivera-Soto R, Gray SJ. Viral expression cassette elements to enhance transgene target specificity and expression in gene therapy. Discov Med. 2015;19(102):49-57.

26. Schepelmann S, Springer CJ. Viral vectors for gene-directed enzyme prodrug therapy. Curr Gene Ther. 2006;6(6):647-670.

27. Srivastava A. Gene therapy with viral vectors: the hope, the problems, and the solution. J Hematother Stem Cell Res. 2001;10(2): 321-322.

28. Flannery JG, Visel M. Adeno-associated viral vectors for gene therapy of inherited retinal degenerations. Methods Mol Biol. 2013;935:351-369.

29. Almarza D, Bussadori G, Navarro M, et al. Risk assessment in skin gene therapy: viral-cellular fusion transcripts generated by proviral transcriptional read-through in keratinocytes transduced with selfinactivating lentiviral vectors. Gene Ther. 2011;18(7):674-681.

30. Kurian KM, Watson CJ, Wyllie AH. Retroviral vectors. Mol Pathol. 2000;53(4):173-176.

31. David RM, Doherty AT. Viral vectors: the road to reducing genotoxicity. Toxicol Sci. 2017;155(2):315-325.

32. Favre D, Provost N, Blouin V, et al. Immediate and long-term safety of recombinant adeno-associated virus injection into the nonhuman primate muscle. Mol Ther. 2001;4(6):559-566.

33. Naso MF, Tomkowicz B, Perry WL 3rd, Strohl WR. Adenoassociated virus (AAV) as a vector for gene therapy. BioDrugs. 2017;31(4):317-334.

34. McClements ME, MacLaren RE. Adeno-associated virus (AAV) dual vector strategies for gene therapy encoding large transgenes. Yale J Biol Med. 2017;90(4):611-623.

35. Yan Z, Zak R, Zhang Y, Engelhardt JF. Inverted terminal repeat sequences are important for intermolecular recombination and circularization of adeno-associated virus genomes. $J$ Virol. 2005;79(1):364-379.

36. Wang D, Tai PWL, Gao G. Adeno-associated virus vector as a platform for gene therapy delivery. Nat Rev Drug Discov. 2019; 18(5):358-378.

37. Maurer AC, Pacouret S, Cepeda Diaz AK, Blake J, AndresMateos E, Vandenberghe LH. The assembly-activating protein promotes stability and interactions between AAV's viral proteins to nucleate capsid assembly. Cell Rep. 2018;23(6):1817-1830.

38. Earley LF, Powers JM, Adachi K, et al. Adeno-associated virus (AAV) assembly-activating protein is not an essential requirement for capsid assembly of AAV serotypes 4, 5, and 11. J Virol. 2017;91(3):e01980-16. 
39. Van Vliet KM, Blouin V, Brument N, Agbandje-McKenna M, Snyder RO. The role of the adeno-associated virus capsid in gene transfer. Methods Mol Biol. 2008;437:51-91.

40. Henckaerts E, Linden RM. Adeno-associated virus: a key to the human genome? Future Virol. 2010;5(5):555-574.

41. Herrmann AK, Große S, Börner K, et al. Impact of the assemblyactivating protein on molecular evolution of synthetic adenoassociated virus capsids. Hum Gene Ther. 2019;30(1):21-35.

42. Grimm D, Buning H. Small but increasingly mighty: latest advances in AAV vector research, design, and evolution. Hum Gene Ther. 2017;28(11):1075-1086.

43. Trapani I. Adeno-associated viral vectors as a tool for large gene delivery to the retina. Genes (Basel). 2019;10(4):E287.

44. Chang YX, Zhao Y, Pan S, et al. Intramuscular injection of adenoassociated virus encoding human neurotrophic factor 3 and exercise intervention contribute to reduce spasms after spinal cord injury. Neural Plast. 2019;2019:3017678.

45. Giannakopoulos A, Quiviger M, Stavrou E, et al. Efficient episomal gene transfer to human hepatic cells using the pFAR4-S/ MAR vector. Mol Biol Rep. 2019;46(3):3203-3211.

46. Falese L, Sandza K, Yates B, et al. Strategy to detect pre-existing immunity to AAV gene therapy. Gene Ther. 2017;24(12): 768-778.

47. Goins WF, Wolfe D, Krisky DM, et al. Delivery using herpes simplex virus: an overview. Methods Mol Biol. 2004;246: 257-299.

48. Colella P, Ronzitti G, Mingozzi F. Emerging issues in AAVmediated in vivo gene therapy. Mol Ther Methods Clin Dev. 2018;8:87-104.

49. Mingozzi F, Meulenberg JJ, Hui DJ, et al. AAV-1-mediated gene transfer to skeletal muscle in humans results in dose-dependent activation of capsid-specific T cells. Blood. 2009;114(10): 2077-2086.

50. Lebherz C, Maguire A, Tang W, Bennett J, Wilson JM. Novel AAV serotypes for improved ocular gene transfer. $J$ Gene Med. 2008;10(4):375-382.

51. Ojala DS, Amara DP, Schaffer DV. Adeno-associated virus vectors and neurological gene therapy. Neuroscientist. 2015;21(1): 84-98.

52. Sen D, Balakrishnan B, Gabriel N, et al. Improved adenoassociated virus (AAV) serotype 1 and 5 vectors for gene therapy. Sci Rep. 2013;3:1832.

53. Salganik M, Hirsch ML, Samulski RJ. Adeno-associated virus as a mammalian DNA vector. Microbiol Spectr. 2015;3(4): $1-21$.

54. Gao GP, Alvira MR, Wang L, Calcedo R, Johnston J, Wilson JM. Novel adeno-associated viruses from rhesus monkeys as vectors for human gene therapy. Proc Natl Acad Sci US A. 2002;99(18): 11854-11859.

55. Kattenhorn LM, Tipper CH, Stoica L, et al. Adeno-associated virus gene therapy for liver disease. Hum Gene Ther. 2016; 27(12):947-961.

56. Collins DE, Reuter JD, Rush HG, Villano JS. Viral vector biosafety in laboratory animal research. Comp Med. 2017;67(3): 215-221.
57. Dismuke DJ, Tenenbaum L, Samulski RJ. Biosafety of recombinant adeno-associated virus vectors. Curr Gene Ther. 2013;13(6): 434-452.

58. Dupont F. Risk assessment of the use of autonomous parvovirusbased vectors. Curr Gene Ther. 2003;3(6):567-582.

59. Mingozzi F, High KA. Immune responses to AAV vectors: overcoming barriers to successful gene therapy. Blood. 2013;122(1): 23-36.

60. Hareendran S, Balakrishnan B, Sen D, Kumar S, Srivastava A, Jayandharan GR. Adeno-associated virus (AAV) vectors in gene therapy: immune challenges and strategies to circumvent them. Rev Med Virol. 2013;23(6):399-413.

61. Russell DW, Grompe M. Adeno-associated virus finds its disease. Nat Genet. 2015;47(10):1104-1105.

62. Cao L, During M, Xiao W. Replication competent helper functions for recombinant AAV vector generation. Gene Ther. 2002; 9(18):1199-1206.

63. Wold WS, Toth K. Adenovirus vectors for gene therapy, vaccination and cancer gene therapy. Curr Gene Ther. 2013;13(6): 421-433.

64. Davison AJ, Benko M, Harrach B. Genetic content and evolution of adenoviruses. J Gen Virol. 2003;84(Pt 11):2895-2908.

65. Khare R, Chen CY, Weaver EA, Barry MA. Advances and future challenges in adenoviral vector pharmacology and targeting. Curr Gene Ther. 2011;11(4):241-258.

66. Campos SK, Barry MA. Current advances and future challenges in adenoviral vector biology and targeting. Curr Gene Ther. 2007; 7(3):189-204.

67. Jager L, Ehrhardt A. Emerging adenoviral vectors for stable correction of genetic disorders. Curr Gene Ther. 2007;7(4):272-283.

68. Ahi YS, Bangari DS, Mittal SK. Adenoviral vector immunity: its implications and circumvention strategies. Curr Gene Ther. 2011; 11(4):307-320.

69. Schaack J. Adenovirus vectors deleted for genes essential for viral DNA replication. Front Biosci. 2005;10:1146-1155.

70. Alba R, Bosch, Chillon M. Gutless adenovirus: last-generation adenovirus for gene therapy. Gene Ther. 2005;12(suppl 1): S18-S27.

71. Morsy MA, Caskey CT. Expanded-capacity adenoviral vectorsthe helper-dependent vectors. Mol Med Today. 1999;5(1):18-24.

72. Lowenstein PR, Mandel RJ, Xiong WD, Kroeger K, Castro MG. Immune responses to adenovirus and adeno-associated vectors used for gene therapy of brain diseases: the role of immunological synapses in understanding the cell biology of neuroimmune interactions. Curr Gene Ther. 2007;7(5):347-360.

73. Zhang Y, Bergelson JM. Adenovirus receptors. J Virol. 2005; 79(19):12125-121231.

74. Hamada K, Desaki J, Nakagawa K, et al. Carrier cell-mediated delivery of a replication-competent adenovirus for cancer gene therapy. Mol Ther. 2007;15(6):1121-1128.

75. Goyvaerts $C$, Breckpot $K$. The journey of in vivo virus engineered dendritic cells from bench to bedside: a bumpy road. Front Immunol. 2018;9:2052.

76. Zhang C, Zhou D. Adenoviral vector-based strategies against infectious disease and cancer. Hum Vaccin Immunother. 2016; 12(8):2064-2074. 
77. Choi Y, Chang J. Viral vectors for vaccine applications. Clin Exp Vaccine Res. 2013;2(2):97-105.

78. Chuah MK, Collen D, Vanden Driessche T. Biosafety of adenoviral vectors. Curr Gene Ther. 2003;3(6):527-543.

79. Gregory SM, Nazir SA, Metcalf JP. Implications of the innate immune response to adenovirus and adenoviral vectors. Future Virol. 2011;6(3):357-374.

80. Rosewell A, Vetrini F, Ng P. Helper-dependent adenoviral vectors. J Genet Syndr Gene Ther. 2011;(suppl 5):001.

81. Liu J, Saghizadeh M, Tuli SS, et al. Different tropism of adenoviruses and adeno-associated viruses to corneal cells: implications for corneal gene therapy. Mol Vis. 2008;14:2087-2096.

82. Mosier DE. Introduction for "safety considerations for retroviral vectors: a short review." Applied Biosaf. 2004;9(2):68-75.

83. Günzburg WH, Fleuchaus A, Saller R, Salmons B. Retroviral vector targeting for gene therapy. Cytokines Mol Ther. 1996; 2(3):177-184.

84. Weiss RA, Tailor CS. Retrovirus receptors. Cell. 1995;82(4): 531-533.

85. Vile RG, Russell SJ. Retroviruses as vectors. Br Med Bull. 1995; 51(1):12-30.

86. Vogt PK. Retroviral oncogenes: a historical primer. Nat Rev Cancer. 2012;12(9):639-648.

87. Maeda N, Fan H, Yoshikai Y. Oncogenesis by retroviruses: old and new paradigms. Rev Med Virol. 2008;18(6):387-405.

88. Mansky LM. Retrovirus mutation rates and their role in genetic variation. J Gen Virol. 1998;79(Pt 6):1337-1345.

89. Doi K, Takeuchi Y. Gene therapy using retrovirus vectors: vector development and biosafety at clinical trials. Uirusu. 2015;65(1): 27-36.

90. Robbins PD, Ghivizzani SC. Viral vectors for gene therapy. Pharmacol Ther. 1998;80(1):35-47.

91. Smith AE. Viral vectors in gene therapy. Annu Rev Microbiol. 1995;49:807-838.

92. Anson DS. The use of retroviral vectors for gene therapy-what are the risks? A review of retroviral pathogenesis and its relevance to retroviral vector-mediated gene delivery. Genet Vaccines Ther. 2004;2(1):9.

93. Romano G. Development of safer gene delivery systems to minimize the risk of insertional mutagenesis-related malignancies: a critical issue for the field of gene therapy. ISRN Oncol. 2012; 2012:616310.

94. Romano G, Marino IR, Pentimalli F, Adamo V, Giordano A. Insertional mutagenesis and development of malignancies induced by integrating gene delivery systems: implications for the design of safer gene-based interventions in patients. Drug News Perspect. 2009;22(4):185-196.

95. Lundstrom K. RNA viruses as tools in gene therapy and vaccine development. Genes (Basel). 2019;10(3):E189.

96. Liu YP, Berkhout B. HIV-1-based lentiviral vectors. Methods Mol Biol. 2014;1087:273-284.

97. Ramezani A, Hawley RG. Overview of the HIV-1 lentiviral vector system. Curr Protoc Mol Biol. 2002; Chapter 16:Unit 16.21.

98. Hall S, Agrawal DK. Delivery of viral vectors for gene therapy in intimal hyperplasia and restenosis in atherosclerotic swine. Drug Deliv Transl Res. 2018;8(4):918-927.
99. Merten OW, Hebben M, Bovolenta C. Production of lentiviral vectors. Mol Ther Methods Clin Dev. 2016;3:16017.

100. Nienhuis AW, Persons DA. Development of gene therapy for thalassemia. Cold Spring Harb Perspect Med. 2012;2(11).

101. Cartier N, Hacein-Bey-Abina S, Bartholomae CC, et al. Lentiviral hematopoietic cell gene therapy for X-linked adrenoleukodystrophy. Methods Enzymol. 2012;507:187-198.

102. Sessa M, Lorioli L, Fumagalli F, et al. Lentiviral haemopoietic stem-cell gene therapy in early-onset metachromatic leukodystrophy: an ad-hoc analysis of a non-randomised, open-label, phase 1/2 trial. Lancet. 2016;388(10043):476-487.

103. Singh S, Khan I, Khim S, et al. Safe and effective gene therapy for murine Wiskott-Aldrich syndrome using an insulated lentiviral vector. Mol Ther Methods Clin Dev. 2017;4:1-16.

104. Zhang C, Liu J, Zhong JF, Zhang X. Engineering CAR-T cells. Biomark Res. 2017;5:22.

105. Levine BL, Miskin J, Wonnacott K, Keir C. Global manufacturing of CAR T cell therapy. Mol Ther Methods Clin Dev. 2017;4: 92-101.

106. Milone MC, O'Doherty U. Clinical use of lentiviral vectors. Leukemia. 2018;32(7):1529-1541.

107. Sandoval Rodríguez AS, Salazar Montes AM, Armendáriz-Borunda J. [Viral vectors in gene therapy. advantages of the adenoassociated vectors]. Rev Gastroenterol Mex. 2005;70(2): 192-202.

108. Palù G, Parolin C, Takeuchi Y, Pizzato M. Progress with retroviral gene vectors. Rev Med Virol. 2000;10(3):185-202.

109. Schlimgen R, Howard J, Wooley D, et al. Risks associated with lentiviral vector exposures and prevention strategies. J Occup Environ Med. 2016;58(12):1159-1166.

110. Goins WF, Huang S, Cohen JB, Glorioso JC. Engineering HSV-1 vectors for gene therapy. Methods Mol Biol. 2014; 1144:63-79.

111. Burton EA, Fink DJ, Glorioso JC. Gene delivery using herpes simplex virus vectors. DNA Cell Biol. 2002;21(12):915-936.

112. Efstathiou S, Minson AC. Herpes virus-based vectors. $\mathrm{Br}$ Med Bull. 1995;51(1):45-55.

113. Manservigi R, Argnani R, Marconi P. HSV recombinant vectors for gene therapy. Open Virol J. 2010;4:123-156.

114. De Silva S, Bowers WJ. Targeting the central nervous system with herpes simplex virus / Sleeping Beauty hybrid amplicon vectors. Curr Gene Ther. 2011;11(5):332-340.

115. Lachmann R. Herpes simplex virus-based vectors. Int J Exp Pathol. 2004;85(4):177-190.

116. Koelle DM, Corey L. Recent progress in herpes simplex virus immunobiology and vaccine research. Clin Microbiol Rev. 2003; 16(1):96-113.

117. O'Reilly M, Shipp A, Rosenthal E, et al. NIH oversight of human gene transfer research involving retroviral, lentiviral, and adeno-associated virus vectors and the role of the NIH recombinant DNA advisory committee. Methods Enzymol. 2012;507: 313-335.

118. Lo B, Grady D. Strengthening institutional review board review of highly innovative interventions in clinical trials. JAMA. 2009; 302(24):2697-2698. 
119. Institute of Medicine. Oversight and Review of Clinical Gene Transfer Protocols: Assessing the Role of the Recombinant DNA Advisory Committee. Washington, DC: National Academies Press; 2014.

120. Cornetta K, Smith FO. Regulatory issues for clinical gene therapy trials. Hum Gene Ther. 2002;13(10):1143-1149.

121. O'Reilly M, Jambou R, Rosenthal E, et al. The national institutes of health oversight of human gene transfer research: enhancing science and safety. Adv Exp Med Biol. 2015;871:31-47.

122. Raper S. An artless tale: challenges faced in clinical research. Food Drug Law J. 2016;71(1):59-104.

123. Grilley BJ, Gee AP. Gene transfer: regulatory issues and their impact on the clinical investigator and the good manufacturing production facility. Cytotherapy. 2003;5(3):197-207.

124. National Institutes of Health (NIH), D.o.H.a.H.S., NIH guidelines for research involving recombinant or synthetic nucleic acid molecules. 2019(April 2019):149.

125. National Institutes of Health Office of Science Policy. From emerging to emerged: streamlining gene therapy oversight. 2018. https://osp.od.nih.gov/2018/08/16/streamlining-gene-ther apy-oversight/. Accessed January 3, 2020.
126. Bubela T, Boch R, Viswanathan S. Recommendations for regulating the environmental risk of shedding for gene therapy and oncolytic viruses in Canada. Front Med (Lausanne). 2019;6:58.

127. Schenk-Braat EA, van Mierlo MM, Wagemaker G, Bangma $\mathrm{CH}$, Kaptein LC. An inventory of shedding data from clinical gene therapy trials. J Gene Med. 2007;9(10): 910-921.

128. Blind JE ME, Campbell KJ. Viral-mediated gene therapy and genetically modified therapeutics: a primer on biosafety handling for the health-system pharmacist. Am J Health Syst Pharm. 2019;76(11):795-802.

129. Keeler AM, Flotte TR. Recombinant adeno-associated virus gene therapy in light of luxturna (and zolgensma and glybera): where are we, and how did we get here? Annu Rev Virol. 2019; 6(1):601-621.

130. Capitini CM. CAR-T immunotherapy: how will it change treatment for acute lymphoblastic leukemia and beyond? Exp Opin Orphan Drugs. 2018;6(10):563-566.

131. Zolgensma - one-time gene therapy for spinal muscular atrophy. Med Lett Drugs Ther. 2019;61(1577):113-114. 